\title{
Involvement of MAP3K7 in FMD2 and CSCF, delineation of genotype/phenotype correlations.
}

Geeske van Woerden ${ }^{1}$, Richelle Senden ${ }^{1}$, Charlotte de Konink ${ }^{1}$, Rossella Avagliano Trezza ${ }^{1}$, anwar baban $^{2}$, Jennifer Bassetti ${ }^{3}$, Yolande Van Bever ${ }^{1}$, Lynne Bird ${ }^{4}$, Bregje van Bon ${ }^{5}$, Alice Brooks ${ }^{1}$, Qiaoning Guan ${ }^{6}$, Eric Klee ${ }^{7}$, Carlo Marcelis ${ }^{8}$, Joel Morales-Rosado ${ }^{7}$, Lisa Schimmenti $^{7}$, Amy Shikany ${ }^{6}$, Paulien Terhal ${ }^{9}$, Kathryn Weaver ${ }^{6}$, Marja Wessels ${ }^{10}$, Hester van Wieringen ${ }^{11}$, Anna Hurst ${ }^{12}$, Catherine Gooch ${ }^{12}$, Marco Tartaglia ${ }^{13}$, Marcello Niceta ${ }^{13}$, Ype Elgersma ${ }^{1}$, and Serwet Demirdas ${ }^{1}$

${ }^{1}$ Erasmus MC

${ }^{2}$ Bambino Gesù Children's Hospital

${ }^{3}$ Weill Cornell Medicine

${ }^{4} \mathrm{UCSD}$

${ }^{5}$ Nijmegen

${ }^{6}$ Cincinnatti Children's Hospital Medical Center Division of Infectious Diseases

${ }^{7}$ Mayo Clinic

${ }^{8}$ Radboudumc

${ }^{9}$ University Medical Center Utrecht

${ }^{10}$ Erasmus University Rotterdam

${ }^{11}$ Sint Antonius Hospital Surgery

${ }^{12}$ Department of Genetics, University of Alabama at Birmingham, Birmingham, Alabama, USA

${ }^{13}$ Bambino Gesù Children's Hospital, IRCCS

November 20, 2021

\begin{abstract}
Mitogen-Activated Protein 3 Kinase 7 (MAP3K7, MIM 602614) encodes the ubiquitously expressed transforming growth factor $\beta$ (TGF- $\beta$ )-activated kinase 1 (TAK1), which plays a crucial role in many cellular processes. Variants in the MAP3K7 gene have been linked to 2 distinct disorders: frontometaphyseal dysplasia type 2 (FMD2, MIM \#617137) and cardiospondylocarpofacial syndrome (CSCF, MIM \#157800). The fact that different variants can induce 2 distinct phenotypes suggests a phenotype/genotype correlation, but no side-by-side comparison has been done thus far to confirm this. Here we significantly expand the cohort and the description of clinical phenotypes for individuals with CSCF and FMD2 who carry variants in MAP3K7. We show that in contrast to FMD2-causing variants, CSCF-causing variants in MAP3K7 have a loss-of-function effect. Additionally, patients with pathogenic variants in MAP3K7 are at risk for cardiac disease, have symptoms associated with connective tissue disease and we show overlap in clinical phenotypes of CSCF with Noonan syndrome. Together, we provide evidence for a molecular fingerprint of FMD2- versus CSCF-causing MAP3K7 variants and conclude that variants in MAP3K7 should be considered in the differential diagnosis of patients with syndromic congenital cardiac defects and/or cardiomyopathy, syndromic connective tissue disorders and in the differential diagnosis of Noonan syndrome.
\end{abstract}

Involvement of MAP3K7 in FMD2 and CSCF, delineation of genotype/phenotype correlations. 
Geeske M. van Woerden ${ }^{1,2,3 \# *}$, Richelle Senden ${ }^{2 \#}$, Charlotte de Konink ${ }^{1,3}$, Rossella Avagliano Trezza ${ }^{1,3}$, Anwar Baban $^{4}$, Jennifer Alisha Bassetti ${ }^{5}$, Yolande van Bever ${ }^{2,3}$, Lynne M. Bird ${ }^{6}$, Bregje W. van Bon ${ }^{7}$, Alice S. Brooks ${ }^{2,3}$, Qiaoning Guan ${ }^{8,9}$, Eric W. Klee ${ }^{10,11}$, Carlo Marcelis ${ }^{7}$, Joel Morales Rosado ${ }^{10,11,12}$, Lisa A. Schimmenti $^{13}$, Amy R Shikany ${ }^{14}$, Paulien A Terhal ${ }^{15}$, K. Nicole Weaver ${ }^{8,9}$, Marja W. Wessels ${ }^{2}$, Hester van Wieringen ${ }^{16}$, Anna C Hurst ${ }^{17}$, Catherine F Gooch ${ }^{17}$, Marco Tartaglia ${ }^{\circ}$, Marcello Niceta ${ }^{\text {os }}$, Ype Elgersma ${ }^{\text {abc }}$ and Serwet Demirdas ${ }^{\mathrm{b} *}$

${ }^{1}$ Department of Neuroscience, Erasmus Medical Center, Rotterdam, the Netherlands.

${ }^{2}$ Department of Clinical Genetics, Erasmus Medical Centre, Rotterdam, the Netherlands.

${ }^{3}$ The ENCORE Expertise Center for Neurodevelopmental Disorders, Erasmus Medical Center, Rotterdam, the Netherlands.

${ }^{4}$ Pediatric Cardiology and Cardiac Arrhythmias Unit, Bambino Gesù Children's Hospital, IRCCS, Rome, Italy.

${ }^{5}$ Division of Medical Genetics, Department of Pediatrics, Weill Cornell Medicine, New York, NY, USA.

${ }^{6}$ Department of Pediatrics, University of California, San Diego; Division of Genetics/Dysmorphology, Rady Children's Hospital San Diego, San Diego, California, USA.

${ }^{7}$ Department of Human Genetics, Radboud University Medical Center, Nijmegen, The Netherlands.

${ }^{8}$ Division of Human Genetics, Cincinnati Children's Hospital Medical Center, Cincinnati, OH, USA.

${ }^{9}$ Department of Pediatrics, University of Cincinnati College of Medicine, Cincinnati, Ohio, USA.

${ }^{10}$ Center for Individualized Medicine, Mayo Clinic, Rochester, MN, USA.

${ }^{11}$ Department of Quantitative Health Sciences, Mayo Clinic, Rochester, MN, USA

${ }^{12}$ Department of Clinical Genomics, Mayo Clinic, Rochester, MN, USA.

${ }^{13}$ Department of Otorhinolaryngology Head and Neck Surgery, Ophthalmology, Clinical Genomics, and Biochemistry and Molecular Biology, Mayo Clinic, Rochester, MN, USA.

${ }^{14}$ The Heart Institute, Cincinnati Children's Hospital Medical Center, Cincinnati, Ohio, USA.

${ }^{15}$ Department of Genetics, University Medical Centre Utrecht, Utrecht, the Netherlands.

${ }^{16}$ Department of Pediatrics, St. Antonius hospital, Nieuwegein, the Netherlands.

${ }^{17}$ Department of Genetics, University of Alabama at Birmingham, Birmingham, Alabama, USA.

${ }^{18}$ Genetics and Rare Diseases Research Division, Ospedale Pediatrico Bambino Gesù, IRCCS, Rome, Italy.

${ }^{19}$ Department of Pediatrics, Sapienza University, Rome, Italy.

\# These authors contributed equally

*Corresponding authors

S. Demirdas, MD PhD (phone: +31 10 7036915; email: s.demirdas@erasmusmc.nl) and G.M. van Woerden, PhD (phone: +31 10 7032695; email: g.vanwoerden@erasmusmc.nl)

\section{Funding}

EWK, JMR, LAS are supported by the Center for Individualized Medicine, Mayo Clinic.

Part of the work of AB, MN and MT was supported by funding from the Italian Ministry of Health (CCR2017-23669081, RCR-2020-23670068_001 to MT) and Italian Ministry of Research (FOE 2019, 2020 Sviluppo di protocolli innovativi e applicazione di nuovi strumenti -omici nei pazienti orfani di diagnosi to MT). GMvW is funded by NWO-VIDI (016.Vidi.188.014) 


\begin{abstract}
Mitogen-Activated Protein 3 Kinase 7 (MAP3K7, MIM 602614) encodes the ubiquitously expressed transforming growth factor $\beta$ (TGF- $\beta$ )-activated kinase 1 (TAK1), which plays a crucial role in many cellular processes. Variants in the MAP3K7 gene have been linked to 2 distinct disorders: frontometaphyseal dysplasia type 2 (FMD2, MIM \#617137) and cardiospondylocarpofacial syndrome (CSCF, MIM \#157800). The fact that different variants can induce 2 distinct phenotypes suggests a phenotype/genotype correlation, but no side-by-side comparison has been done thus far to confirm this. Here we significantly expand the cohort and the description of clinical phenotypes for individuals with CSCF and FMD2 who carry variants in MAP3K 7 . We show that in contrast to FMD2-causing variants, CSCF-causing variants in MAP3K 7 have a loss-of-function effect. Additionally, patients with pathogenic variants in MAP3K 7 are at risk for cardiac disease, have symptoms associated with connective tissue disease and we show overlap in clinical phenotypes of CSCF with Noonan syndrome. Together, we provide evidence for a molecular fingerprint of FMD2- versus CSCF-causing MAP3K 7 variants and conclude that variants in MAP3K 7 should be considered in the differential diagnosis of patients with syndromic congenital cardiac defects and/or cardiomyopathy, syndromic connective tissue disorders and in the differential diagnosis of Noonan syndrome.
\end{abstract}

Keywords: MAP3K7, frontometaphyseal dysplasia type 2, cardiospondylocarpofacial syndrome, Noonan Syndrome

\title{
Introduction
}

The MAP3K 7 gene (Mitogen-Activated Protein 3 Kinase 7) encodes transforming growth factor $\beta$ (TGF$\beta$ )-activated kinase 1 (TAK1), which plays a vital role in innate and adaptive immunity by regulating inflammatory responses and regulating cell differentiation, cell survival and apoptosis

(Dai et al., 2012; Yu et al., 2014). Variants in MAP3K7 have been associated with two autosomal dominant conditions; frontometaphyseal dysplasia type 2 (FMD2) caused by recurrent gain-of-function variants

(Wade et al., 2016), and cardiospondylocarpofacial syndrome (CSCF) caused by individual variants most often considered to induce loss-of-function

(Le Goff et al., 2016).

FMD is a progressive skeletal dysplasia characterized by sclerosis of the skull, joint contractures and undermodeled long bones, scoliosis and prominent supraorbital ridges. Other features include hypertelorism, down-slanting palpebral fissures, broad nasal bridge, full cheeks, micrognathia, hydronephrosis, cleft palate, hearing loss, ulnar deviation of the hands, camptodactyly, wrist contractures, long fingers, structural cardiac defect and keloid scars (Costantini et al., 2018). FMD is caused by gain-of-function variants in FLNA, TAB2 and MAP3K 7

(Robertson, 2004; Giuliano et al., 2005; Wade et al., 2016; 2017). Most individuals have normal intellect, although intellectual disability has been reported

(Basart et al., 2015; Wade et al., 2016). In total, 19 FMD2 patients carrying variants in MAP3K 7 have been described in literature (Wade et al., 2017; Costantini et al., 2018). A recurrent variant was identified in 16 patients (c.1454C>T (p.Pro485Leu)), and the other three described variants causing the FMD2 phenotype are c.208G $>$ C (p.Glu70Gln), c.299T $>$ A (p.Val100Glu) and c.502G $>$ C (p.Gly168Arg). This recurrent variant leads to a severe skeletal phenotype, keloid scarring, and (in 3 out of 16 patients) intellectual disability (ID). The three patients with the infrequent missense variants show a milder phenotype consistent with FMD, without ID (Costantini et al., 2018).

CSCF was first described in 1966 as a new syndrome and autosomal dominant inheritance was suggested (Forney et al., 1966). The initial report describes a mother and two of her four daughters with congenital mitral valve insufficiency, skeletal malformations (fusion of cervical vertebrae/tarsal bones/carpal bones, phalangeal shortening), conductive deafness and short stature. The hearing loss resulted from fusion of the stapes to the round window and was improved by surgical correction. In 2010, two additional patients with 
an overlapping phenotype were reported introducing the name CSCF for the first time (Sousa et al., 2010). However, it was not until 2016 that the gene MAP3K 7 was identified as the cause of CSCF by Le Goff et al.

(Le Goff et al., 2016) who described 6 patients with CSCF phenotype and a causative heterozygous missense variant in the gene: three sporadic cases and one family (a father and two sons). Morlino et al. summarize these patients in their publication, adding a twelfth novel patient with CSCF due to a de novo variant in the MAP3K 7 gene (Morlino et al., 2018). In 2020, the same group published functional studies on patient fibroblasts demonstrating that CSCF is caused by loss-of-function variants in the gene through the TGF $\beta$ pathway (Micale et al., 2020). Interestingly, intellectual disability (ID) has been described in FMD2, but not in CSCF.

Here, we expand the cohort of individuals with CSCF and FMD2 carrying variants in MAP3K 7 , describing 14 novel patients with CSCF and 2 novel patients with FMD2 (one with the recurrent variant and severe ID). Based on the phenotype of the patients we suggest that both CSCF and FMD2 are connective tissue diseases. We describe severe congenital left sided cardiac defects in CSCF patients and we hypothesize an overlap of CSCF patients with the Noonan syndrome (NS) (OMIM 163950) phenotype. Additionally, we provide for the first time a side-by-side comparison of the biochemical effects of missense variants in MAP3K 7 for CSCF and FMD2, aiming to find a molecular fingerprint to distinguish between the two disorders.

\section{Methods}

PatientsSixteen patients with novel variants in the $M A P 3 K^{7}$ gene were included in this clinical observation with functional laboratory analysis of the variants. Patients were identified in the clinical setting with trio whole-exome sequencing. Patients from five countries ( 7 medical centers in total) were identified using Genematcher (Sobreira et al., 2015). Written consent was obtained from adult patients and parents of pediatric patients for participation in the study and for publishing photographs.

\section{Clinical observations}

Cross-sectional clinical assessment (medical history, physical examination, variant classification and (if consented) photography) was performed by the treating physician to complete a detailed chart of phenotypic features. If available, radiologic, and cardiac analysis outcomes were collected from the medical file. All data were entered in a pseudo-anonymized digital database. The descriptive data were analyzed and the results are presented in the manuscript text and Table $\mathbf{1}$.

\section{Ethical considerations}

The study was conducted according to the principles of the Declaration of Helsinki (10th version, October 2013, see for the most recent version: www.wma.net (World Medical Association 2013)) and in accordance with the Dutch Medical Research Involving Human Subjects Act (WMO). Approval from the medical ethics comity of the Erasmus Medical Center was given for conducting our retrospective case-series study in these patients.

\section{Laboratory assessments}

Constructs

The MAP3K $\eta^{W T}$ (NM_003188.4) cDNA was obtained from the human brain library by PCR (Phusion high fidelity, Thermo Fisher) using the following primers: FW 5'-GTCTCACCCGGATTGTCC; RV 5'AATGTAACGGTCCCAGAG. To be able to clone this sequence into our dual promotor expression vector (Proietti Onori et al., 2018), the fragment was tagged with restriction enzymes AscI; PacI by PCR (Phusion high fidelity, Thermo Fisher) using primers: FW 5'- GAATTGCGGCGCGCCACCATGTCTACAGCCTCTGCC; RV 5'- GAATCCTTAATTAATCATGAAGTGCCTTGTCG. The point mutations were introduced with site-directed mutagenesis (Phusion high fidelity, Thermo Fisher) using the following primers: MAP3K7 c.143G $>$ A (p.Gly48Glu), FW 5'-GGAAGAGGAGCCTTTGAAGTTGTTTGCAAAGCT and RV 5'-AGCTTTGCAAACAACTTCAAAGGCTCCTCTTCC; MAP3K' $\quad$ c. $\quad 208 \mathrm{G}>\mathrm{C}$ 
(p.Glu70Gln), FW 5'- TAAACAAATAGAAAGTGAATCTCAGAGGAAAGCGTTTATTGTAGA and RV 5'- TCTACAATAAACGCTTTCCTCTGAGATTCACTTTCTATTTGTTTA; MAP3K 7 c.248G $>$ A (p.Arg83His), FW 5'- GCTTCGGCAGTTATCCCATGTGAACCATCCTAATA and RV 5'- TATTAGGATGGTTCACATGGGATAACTGCCGAAGC; MAP3K 7 c. 299T $>$ A (p.Val100Glu), FW 5'- GGAGCCTGCTTGAATCCAGAGTGTCTTGTGATGGAATAT and RV 5'- ATATTCCATCACAAGACACTCTGGATTCAAGCAGGCTCC; MAP3K 7 c.329G $>$ A (p.Gly110Asp), FW 5'- GATGGAATATGCTGAAGGGGACTCTTTATATAATGTGCTGC and RV 5'-GCAGCACATTATATAAAGAGTCCCCTTCAGCATATTCCATC; $\quad M A P 3 K^{\prime}$ c. $337 \mathrm{~T}>\mathrm{G}$ (p.Tyr113Asp), FW 5'-TGAAGGGGGCTCTTTAGATAATGTGCTGCATGG and RV 5CCATGCAGCACATTATCTAAAGAGCCCCCTTCA; MAP3K 7 c.502G>C (p.Gly168Arg), FW 5'CTTACTGCTGGTTGCAGGGCGGACAGTTCTAAAAATTTG and RV 5'- CAAATTTTTAGAACTGTCCGCCCTGCAACCAGCAGTAAG; MAP3K7c.586A>G (p.Met196Val), FW 5'- GGGAGTGCTGCTTGGGTGGCACCTGAAGTTT and RV 5'-AAACTTCAGGTGCCACCCAAGCAGCACTCCC; MAP3K 7 c.617A $>$ G (p.Tyr206Cys), FW 5'- GAAGTTTTTGAAGGTAGTAATTGCAGTGAAAAATGTGACGTCTTC and RV 5'- GAAGACGTCACATTTTTCACTGCAATTACTACCTTCAAAAACTTC; $M A P 3 K^{\gamma}$ c.616T $>$ G (p.Tyr206Asp), FW 5'- GTTTTTGAAGGTAGTAATGACAGTGAAAAATGTGACG and RV 5'- CGTCACATTTTTCACTGTCATTACTACCTTCAAAAAC; MAP3K'c.721T>G (p.Trp241Gly), FW 5'- CCCAGCTTTCCGAATCATGGGGGCTGTTCATAATGGTAC and RV 5'- GTACCATTATGAACAGCCCCCATGATTCGGAAAGCTGGG; $\quad M A P 3 K$ 7. $1454 \mathrm{C}>\mathrm{T}$ (p.Pro485Leu), FW 5'-ACTACAGCCTCTAGCACTGTGCCCAAACTCCAAAG and RV 5'CTTTGGAGTTTGGGCACAGTGCTAGAGGCTGTAGT. For the in vivoexperiments the 'empty vector' serves as control, consisting of the dual promotor expression vector only (no gene insertion in MCS).

The TAB1 ${ }^{w t}$ (NM_006116.3) cDNA was obtained from human brain library by PCR (Phusion high fidelity, Thermo Fisher) using the following primers: FW 5'-ATGGCGGCGCAGAGGAG and RV 5'- CTACGGTGCTGTCACCAC. This was then also cloned in our dual promotor expression vector, tagging the fragment with restriction enzymes AscI; PacI by PCR (Phusion high fidelity, Thermo Fisher) using primers: FW 5'-GGCGCGCCACCATGGCGGCGCAGAGGAG and RV 5'-TTAATTAACTACGGTGCTGTCACCAC.

\section{HEK293T transfection}

To assess the functional effects of the different MAP3K 7 variants, HEK293T cells were cultured in DMEM/10\% Fetal Calf Serum (FCS)/1\% penicillin/streptomycin in 6-wells plates and transfected with the following constructs: Control vector;MAP3K $\eta^{W T} ; M A P 3 K^{\gamma G 48 E} ; M A P 3 K^{\gamma E 70 Q} ; M A P 3 K^{R 833 H}$;

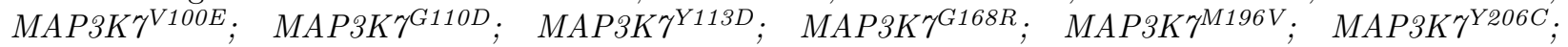
MAP3K $7^{Y 206 D} ; M A P 3 K^{r W 241 G} ; M A P 3 K^{r P 485 L} ; T A B 1^{W T}$. In single construct transfections, 3ug construct per well was transfected. When in co-transfection of the different MAP3K 7 constructs with $T A B 1^{W T}$ , $1.5 \mu \mathrm{g} /$ construct/well was transfected. Constructs for co-transfection were mixed before actual transfection, allowing both constructs to be transfected at the same time. At subconfluency (60-70\%), cells were transfected with PEI according to the manufacturer instructions (Sigma). To avoid toxicity of prolonged exposure to PEI, the medium was refreshed after 4-6 hours. Transfected cells were used to prepare protein lysates for Western blot.

Western blot

The cells were harvested in ice cold PBS, 48-72 hours after transfection. Lysate samples were prepared by homogenizing the harvested HEK293Tcells in lysate buffer (10mM Tris-HCl 6.8, 2.5\% SDS, 2mM EDTA) containing protease inhibitor cocktail 2 (\#P5726, Sigma) and 3 (\#P0044, Sigma) and protease inhibitor (\#P8340, Sigma). Protein concentrations were determined using the BCA kit (Pierce). Final working protein concentrations were adjusted to $1 \mathrm{mg} / \mathrm{ml}$. Western blots were probed with primary antibodies against MAP3K7 (sc-7967, 1:1000, Santa Cruz), phospho-MAP3K7 (Thr187; \#4536, 1:1000, Cel Signalling), ERK1/2 (\#9102, 1:2000, Cell Signaling), phospho-ERK1/2 (\#9101, 1:2000; Cell Signaling), Actin (MAB1501R, 1:20.000, Chemicon), RFP (\#600401379, 1:2000, Rockland), NFKB (sc-514451, 1:1000, Santa Cruz), phospho-NFkb (sc-136548, 1:1000, Santa Cruz), GAPDH (2118S, 1:2000, Cell Signaling), TAB1 
(67020-1-Ig, 1:10.000, Proteintech) and secondary antibodies (goat anti-mouse (\#926-32210) and goat antirabbit (\#926-68021), all 1:15.000, LI-COR). Blots were quantified using LI-COR Odyssey Scanner and Odyssey 3.0 software.

\section{Statistical analysis}

All data were assumed to be normally distributed. Statistical difference between the conditions for the in vitro overexpression experiments was determined using one-way analysis of variance (ANOVA) followed by Bonferroni's post-hoc test for multiple comparisons. For the Western blot analysis, two-tailed unpaired t-test was used (dual comparison). Neuronal migration was analyzed based on the proportion of electroporated cells targeted to the cortical plate at P1 (defined as the most proximal $40 \%$ of dorsoventral distance between the pia and ventricle (first 4 of 10 equally-spaced bins)) or to layer $2 / 3$ of the somatosensory cortex, defined as the proximal $30 \%$ of dorsoventral distance between the proximal $10 \%$ of the cortex (assumed to represent layer 1) and ventricle (bin 2-4 of equally-spaced bins). For neuronal migration analyses, we used a minimum of 3 targeted pups per condition.

\section{Results}

A combined overview of the variants in our cohort and reported in literature are shown in Figure 1 . Patient characteristics and detailed phenotypic description are shown in Table $\mathbf{1}$. If a specific feature was not clearly reported as positive or negative for a patient, this feature was considered as missing data for the respective patient. For this reason, the total number of patients per feature may differ in our results.

\section{Clinical phenotype of cardiospondylocarpofacial syndrome}

Of the fourteen patients with the CSCF phenotype, twelve carriedde novo variants in MAP3K 7 , one female inherited the variant from her father and one female did not inherit the variant from her mother, but father was unavailable for testing. The clinical phenotype and patient characteristics are presented in detail inTable 1. Two adult CSCF patients were included. Figure 2 shows photographs of the patients with CSCF who consented for publication.

\section{Dysmorphic features}

The most frequently recorded dysmorphic features are hypotonic face (6/12 patients), full cheeks $(9 / 13)$, low set ears $(5 / 10)$, posteriorly rotated ears $(7 / 12)$, hypertelorism $(8 / 12)$, ptosis $(8 / 13)$, epicanthal folds (6/12), peri-orbital fullness $(7 / 11)$, anteverted nares $(7 / 11)$, round tipped nose $(9 / 11)$, long philtrum $(7 / 9)$ and widely spaced nipples $(7 / 9)$. Short stature $(<-2$ SDS for age and gender) was observed in $9 / 14$ CSCF patients, ranging from -2.1 SDS to -4 SDS. Other less frequently reported features are listed in Table 1 .

\section{Cardiac features}

Interestingly, CSCF patients showed a wide range of cardiac anomalies. Congenital heart defects that mostly seem to be left sided are reported in 7/13 patients ranging from mitral valve prolapse to hypoplastic left heart syndrome spectrum (Y206C variant, patient 11; Table 1 ). Cardiomyopathy was observed in 4/12 patients (1 hypertrophic and 3 dilated), the most severe in a neonate with the $143 \mathrm{G}>\mathrm{A}$ variant (patient 2 ), who is on the waiting list for heart transplantation.

\section{Musculoskeletal features}

Carpal fusion was assessed in seven patients and found in two of them. Brachydactyly was frequently observed (6/9). Pectus excavatum was seen in 3/11 patients. Joint laxity was a consistent feature in the pediatric patients and reported in 8/10 patients. Scoliosis was reported in two patients and for one of the patients this was described as a Hobbs curve of 35 degrees. Vertebral anomalies were identified in 4/6 patients and involved fusion of vertebrae (also of the odontoid process and anterior arch of C1), small intervertebral distance (thoracic), loss of height of vertebrae and dysmorphic vertebrae. No contractures were described in our CSCF patients.

Neurologic features 
No ID was described in any of the CSCF patients. Autism spectrum disorder, however, was present in $3 / 7$ patients. Other neurologic features that drew attention are hypotonia $(7 / 10)$ and hypoplasia of muscles; right pectoralis hypoplasia in one patient and hypoplasia of the adductor pollicis $(3 / 6)$.

Hearing loss

Five out of eleven patients are reported to have hearing loss: 1 sensorineural with defective inner ear formations, and the other 4 conductive/mixed.

\section{Other features}

One male patient had cryptorchidism. Ten out of 11 patients had feeding difficulties at a young age, frequently needing nasogastric tube feeding for a period of time.

Clinical phenotype frontometaphyseal dysplasia type 2

Only two patients in this cohort have FMD2. Both patients have ade novo variant (one having the recurrent variant). The clinical phenotype and patient characteristics are presented in detail in

Table 1.

Dysmorphic features

The reported dysmorphic features in our two patients are consistent with the FMD2 patients reported in medical literature, including full cheeks, hypertelorism, epicanthal folds and micrognathia (Figure 3 ).

\section{Cardiac features}

Both FMD2 patients had a persistent ductus arteriosus, which was part of a more complex cardiac defect (ventricular septal defect, pulmonary atresia, bicuspid aortic valve with mild stenosis, progressive aortic root dilatation ( $>3$-z-score), persistent left superior vena cava draining into the coronary sinus and ectopic atrial tachycardia) in the patient born at 35 weeks of gestation with the recurrent $\mathrm{P} 485 \mathrm{~L}$ variant. The congenital heart defect in this patient was a major reason for failure to thrive and recurrent admissions in her early months of life.

\section{Musculoskeletal features}

Both FMD2 patients had scoliosis and short stature (-2.2 SDS and -3.1 SDS). Both patients show camptodactyly of their fingers, flexion contractures (elbow with dislocation of the radial head) and carpal fusion. The older male patient has joint laxity. The younger female patient shows flared metaphyses, broad thumbs/fingers osteosclerosis, and partially fused with dysmorphic cervical vertebrae.

\section{Neurologic features}

The patient with the recurrent variant has severe ID. Her brain MRI shows cerebral atrophy, thin corpus callosum and Chiari 1 malformation. She also has hypotonia and is suspected to possibly have muscular atrophy. The other patient has normal intellect and tone, as well as muscular hypoplasia of his hands.

\section{Hearing loss}

The adult patient (with the non-recurrent variant) was shown to have mixed conductive/sensorineural hearing loss. The pediatric female patient with the recurrent variant was reported to also have hearing loss (type not specified). No temporal bone imaging is available for these patients.

\section{Other features}

The adult patient (with the non-recurrent variant) had cryptorchidism and was consulted for ambiguous genitalia as a newborn. The young female patient (with the recurrent variant) had congenital stridor due to laryngo - trachea-bronchomalacia that improved with time and without the need for tracheostomy. Feeding problems are managed by PEG. Both patients showed excessive keloid scarring. 
The differences in clinical features seen in FMD2 versus CSCF patients are thought to be caused by different underlying molecular mechanisms. MAP3Kr variants giving rise to FMD2 are most often recurrent, gain-offunction variants

(Wade et al., 2016), whereas CSCF is frequently caused by non-recurrent missense variants, where for some variants a loss-of-function effect was shown, but the functional effect of other variants remains unknown. Our cohort, in combination with already published patients, allowed us to assess whether there is indeed a molecular fingerprint that can distinguish between FMD2 and CSCF. Since our cohort has only 2 FMD2 patients, we included the previously published FMD2-causing missense variants in $M A P 3 K^{r}$

(Wade et al., 2016).

In searching for a molecular fingerprint, we first assessed the expression levels of the different $M A P 3 K^{r}$ variants upon overexpression in HEK-293T cells. Whereas the variants causing FMD2 showed similar or even a significantly higher expression level compared to MAP3K $7^{\mathrm{WT}}$, all CSCF variants showed significantly lower expression levels compared to MAP3K $7^{\mathrm{WT}}$ (FMD2 variants: one-way ANOVA F[5,25]=6.16, $\mathrm{p}=0.0008 ;$ MAP3K $7^{\mathrm{WT}}$ versus MAP3K $7^{\mathrm{E} 70 \mathrm{Q}}: \mathrm{p}=0.39 ; \mathrm{MAP} 3 \mathrm{~K} 7^{\mathrm{WT}}$ versus MAP3K $7^{\mathrm{V} 100 \mathrm{E}}: \mathrm{p}=0.087 ;$

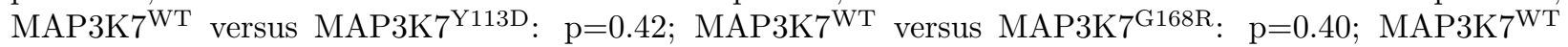
versus MAP3K7 ${ }^{\mathrm{P} 485 \mathrm{~L}}$ : $\mathrm{p}=0.0079$, Dunnett's multiple comparison test; CSCF variants: one-way ANOVA $\mathrm{F}[7,23]=6.39, \mathrm{p}=0.0003 ; \mathrm{MAP} 3 \mathrm{~K} 7^{\mathrm{WT}}$ versus MAP3K $7^{\mathrm{G} 48 \mathrm{E}}: \mathrm{p}=0.004 ;$ MAP3K $^{\mathrm{WT}}$ versus MAP3K ${ }^{\mathrm{R} 83 \mathrm{H}}$ :

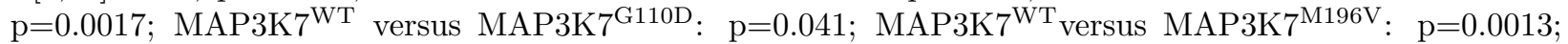

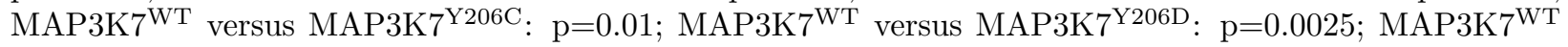
versus MAP3K7W241G: $\mathrm{p}<0.0001$, Dunnett's multiple comparison test ; Figure 4A ). With the exception of MAP3K $7^{\mathrm{W} 241 \mathrm{G}}$, reduced expression levels of the CSCF variants could be normalized to control levels upon co-transfection with TAB1 (one-way ANOVA F[7,54]=4.3, p $<0.001$; MAP3K7 ${ }^{\mathrm{WT}}$ versus MAP3K $7^{\mathrm{G} 48 \mathrm{E}}: \mathrm{p}<0.0001 ;$ MAP3K $^{\mathrm{WT}}$ versus MAP3K $7^{\mathrm{R} 83 \mathrm{H}}: \mathrm{p}=0.86 ;$ MAP3K $^{\mathrm{WT}}$ versus MAP3K7 ${ }^{\mathrm{G} 110 \mathrm{D}}$ : $\mathrm{p}=0.9 ;$ MAP3K $^{\mathrm{WT}}$ versus MAP3K $7^{\mathrm{M} 196 \mathrm{~V}}: \mathrm{p}=0.25 ; \mathrm{MAP}^{\mathrm{K}} 7^{\mathrm{WT}}$ versus MAP3K $^{\mathrm{Y} 206 \mathrm{C}}: \mathrm{p} 0.9 ;$ MAP3K $7^{\mathrm{WT}}$ versus MAP3K $7^{\mathrm{Y} 206 \mathrm{D}}: \mathrm{p}=0.06$; MAP3K $7^{\mathrm{WT}}$ versus MAP3K $7^{\mathrm{W} 241 \mathrm{G}}: \mathrm{p}=0.02$, Dunnett's multiple comparison test; Figure 4B ), indicating that these variants cause instability of the MAP3K7 protein in the absence of TAB1.

Co-expression of $M A P 3 K^{\gamma}$ with $T A B 1$ has been shown to result in $M A P 3 K^{\gamma}$ autophosphorylation at Thr187, as well as a slower migration of the TAB1 band on Western blot (Sakurai et al., 2000). Therefore, we used this assay as a readout for the kinase activity of $M A P 3 K^{\gamma}$. Consistent with literature, co-expression of MAP3K7 ${ }^{\mathrm{WT}}$ with TAB1 resulted in slower migration of the MAP3K7, as well as the TAB1 band on Western blot (Figure 4B ), which corresponded with MAP3K7 autophosphorylation of MAP3K7 at Thr187 (Figure 4C ). In support of previous findings that FMD2 is caused mainly by gain-of-function variants, co-expression of the FMD2-related $M A P 3 K^{7}$ variants with TAB1, all resulted in equal or (a trend towards) increased levels of autophosphorylation of $M A P 3 K^{r} 7$ at Thr187 compared to MAP3K7 ${ }^{\mathrm{WT}}$ (one-way ANOVA $\mathrm{F}[5,42]=5.43, \mathrm{p}=0.0006 ; \mathrm{MAP}_{\mathrm{KK}} 7^{\mathrm{WT}}$ versus MAP3K $7^{\mathrm{E} 70 \mathrm{Q}}: \mathrm{p}=0.9 ; \mathrm{MAP} 3 \mathrm{~K} 7^{\mathrm{WT}}$ versus MAP3K7V100E: $\mathrm{p}=0.4 ;$ MAP3K $7^{\mathrm{WT}}$ versus MAP3K7 $7^{\mathrm{Y} 113 \mathrm{D}}: \mathrm{p}=0.03 ;$ MAP3K $7^{\mathrm{WT}}$ versus MAP3K7 $7^{\mathrm{G} 168 \mathrm{R}}: \mathrm{p}=0.0004 ;$ MAP3K $7^{\mathrm{WT}_{\text {versus }} \text { MAP3K }} 7^{\mathrm{P} 485 \mathrm{~L}}: \mathrm{p}=0.9$, Dunnett's multiple comparison test (Figure 4C )). In contrast, upon co-transfection with TAB1 the majority of the CSCF-related MAP3K7 variants showed significantly reduced pThr187 autophosphorylation levels compared to MAP3K7 ${ }^{\mathrm{WT}}$ (one-way ANOVA F[7,54]=15.9, $\mathrm{p}<0.0001 ;$ MAP3K $7^{\mathrm{WT}}$ versus MAP3K7 ${ }^{\mathrm{G} 48 \mathrm{E}}: \mathrm{p}<0.0001 ; \mathrm{MAP} 3 \mathrm{~K} 7^{\mathrm{WT}}$ versus MAP3K $7^{\mathrm{G} 110 \mathrm{D}}: \mathrm{p}<0.0001 ;$ MAP3K7 ${ }^{\mathrm{WT}}$ versus MAP3K7 ${ }^{\mathrm{M} 196 \mathrm{~V}}: \mathrm{p}<0.0001 ; \mathrm{MAP}^{\mathrm{K}} 7^{\mathrm{WT}}$ versus MAP3K $^{\mathrm{Y} 206 \mathrm{C}}: \mathrm{p}=0.0002 ; \mathrm{MAP} 3 \mathrm{~K} 7^{\mathrm{WT}}$ versus MAP3K $7^{\text {Y206D }}: \mathrm{p}<0.0001$; MAP3K $7^{\mathrm{WT}}$ versus MAP3K $7^{\mathrm{W} 241 \mathrm{G}}: \mathrm{p}<0.0001$, Dunnett's multiple comparison test (Figure 4C )). Interestingly, one CSCF-related $M A P 3 K^{r}$ variant (MAP3K $7^{\mathrm{R} 83 \mathrm{H}}$ ), did not show reduced pThr187 autophosphorylation levels compared to MAP3K $7^{\mathrm{WT}}$ (MAP3K $7^{\mathrm{WT}}$ versus MAP3K7 ${ }^{\mathrm{R} 83 \mathrm{H}}$ : $\mathrm{p}=0.48$, Dunnett's multiple comparison test (Figure $\mathbf{4 C})$ ).

MAP3K7 is known to have several downstream substrates, through which it affects different pathways 
in the cell (Xu and Lei, 2020; Aashaq et al., 2019). Finding that the stability and the autophosphorylation levels seem to distinguish between CSCF and FMD2-related MAP3K 7 variants, we next sought to understand whether there is a difference in substrate regulation between the CSCF and FMD2-related $M A P 3 K$ \% variants. Consistent with the reduced autophosphorylation, we found that the CSCF-related $M A P 3 K^{7}$ variants resulted in reduced phosphorylated $\mathrm{NFkB}$ compared to MAP3K $7^{\mathrm{WT}}$, again with the exception of MAP3K7 $7^{\mathrm{R} 83 \mathrm{H}}$ (one-way ANOVA $\mathrm{F}[7,51]=23.73, \mathrm{p}<0.0001 ; \mathrm{MAP}^{\mathrm{K}} 7^{\mathrm{WT}}$ versus MAP3K7 $^{\mathrm{G} 48 \mathrm{E}}$ : $\mathrm{p}<0.0001$; MAP3K7 $7^{\mathrm{WT}}$ versus MAP3K7 ${ }^{\mathrm{R} 83 \mathrm{H}}$ : $\mathrm{p}=0.66$; MAP3K7 $7^{\mathrm{WT}}$ versus MAP3K7 $7^{\mathrm{G} 110 \mathrm{D}}$ : $\mathrm{p}<0.0001$; MAP3K $7^{\mathrm{WT}}$ versus MAP3K7 ${ }^{\mathrm{M} 196 \mathrm{~V}}: \mathrm{p}<0.0001 ; \mathrm{MAP} \mathrm{K} 7^{\mathrm{WT}}$ versus MAP3K $^{\mathrm{Y} 206 \mathrm{C}}: \mathrm{p}<0.0001 ;$ MAP3K $7^{\mathrm{WT}}$ versus MAP3K7 ${ }^{\mathrm{Y} 206 \mathrm{D}}: \mathrm{p}<0.0001 ;$ MAP3K7 ${ }^{\mathrm{WT}}$ versus MAP3K7 ${ }^{\mathrm{W} 241 \mathrm{G}}: \mathrm{p}<0.0001$, Dunnett's multiple comparison test (Figure 5A )).

As for the FMD2-related MAP3K7 variants, the results were less straightforward. Overall, the variants did not cause increased levels of phosphorylated NFkB, instead most variants showed a trend or significant reduction in phosphorylated NFkB (one-way ANOVA $\mathrm{F}[5,49]=4.11, \mathrm{p}=0.0034$; MAP3K $7^{\mathrm{WT}}$ versus MAP3K7 ${ }^{\text {E70Q }}: \mathrm{p}=0.04$; MAP3K7 $^{\mathrm{WT}}$ versus MAP3K7 ${ }^{\mathrm{V} 100 \mathrm{E}}: \mathrm{p}=0.4 ;$ MAP3K7 $^{\mathrm{WT}}$ versus MAP3K $^{\mathrm{Y} 113 \mathrm{D}}$ : $\mathrm{p}=0.54$; MAP3K $7^{\mathrm{WT}}$ versus MAP3K7 ${ }^{\mathrm{G} 168 \mathrm{R}}: \mathrm{p}=0.018$; MAP3K $7^{\mathrm{WT}}$ versus MAP3K7 $^{\mathrm{P} 485 \mathrm{~L}}: \mathrm{p}=0.74$, Dunnett's multiple comparison test (Figure 5A )). These results indicate that whereas stability and autophosphorylation of MAP3K 7 at Thr187 can potentially be used as molecular fingerprint for distinguishing FMD2 and CSCF variants, not all downstream pathways of MAP3K7 are necessarily differentially affected.

The majority of the CSCF patients showed clinical features in part similar to NS, where the RAS-MAPK pathway is hyperactivated (van der Burgt, 2007; Jorge et al., 2009). We therefore assessed whether the CSCF-related MAP3K7 variants upregulated the RAS-MAPK pathway. However, in contrast to what is normally seen in NS, we found that most of the CSCF-related MAP3K 7 variants resulted in reduced phosho-ERK levels compared to MAP3K7 ${ }^{\mathrm{WT}}$ (one-way ANOVA $\mathrm{F}[7,24]=6.78, \mathrm{p}=0.0002$; MAP3K $7{ }^{\mathrm{WT}}$ versus MAP3K7 ${ }^{\mathrm{G} 48 \mathrm{E}}: \mathrm{p}=0.016$; MAP3K7 ${ }^{\mathrm{WT}}$ versus MAP3K7 ${ }^{\mathrm{G} 110 \mathrm{D}}: \mathrm{p}=0.007$ MAP3K7 $^{\mathrm{WT}}$ versus MAP3K7 $^{\mathrm{M} 196 \mathrm{~V}}$ : $\mathrm{p}=0.005 ;$ MAP3K7 $7^{\mathrm{WT}}$ versus MAP3K7 ${ }^{\mathrm{Y} 206 \mathrm{C}}: \mathrm{p}=0.008 ;$ MAP3K7 ${ }^{\mathrm{WT}}$ versus MAP3K7 $7^{\mathrm{Y} 206 \mathrm{D}}: \mathrm{p}=0.009$; MAP3K7 $7^{\mathrm{WT}}$ versus MAP3K7 ${ }^{\mathrm{W} 241 \mathrm{G}}$ : $\mathrm{p}=0.021$, Dunnett's multiple comparison test (Figure 5B )). Consistent with our previous results, only MAP3K $7^{\mathrm{R} 83 \mathrm{H}}$ behaved similar to MAP3K7 ${ }^{\mathrm{WT}}$ (MAP3K $7^{\mathrm{WT}}$ versus MAP3K7 ${ }^{\mathrm{R} 83 \mathrm{H}}: \mathrm{p}=0.9$, Dunnett's multiple comparison test (Figure 5B )).

\section{Discussion}

In this study, we significantly expand the published cohort of patients with variants in the MAP $3 K^{7}$ gene, causing either CSCF or FMD2 and assess for the first time the genotype-phenotype correlation for MAP3K 7 variants. We show that missense variants in $M A P 3 K^{\prime}$ causing CSCF reduce MAP3K7 protein stability and autophosphorylation at Thr187, whereas MAP3K 7 variants causing FMD2 show normal or enhanced MAP3K7 protein expression and autophosphorylation levels. However, when assessing one of the downstream targets, NFkB, no difference was observed between the variants causing CSCF or FMD2, indicating that further research is required to assess what downstream targets are differentially affected, to further elucidate the precise molecular mechanism underlying CSCF and FMD2.

Using whole exome sequencing (WES) in 5 independent Dutch pediatric patients (from two separate medical centers) suspected to have NS, variants of unknown significance were found in MAP3K7 . Further clinical assessment (detailed physical examination and radiologic assessment of the skeleton) of these patients confirmed the diagnosis of CSCF. To further characterize genotype/phenotype correlations in these patients, we studied 13 children (12 CSCF, 1 FMD2) and 3 adults ( 1 FMD2) with variants in MAP3K 7 . Features of our CSCF patients overlapping NS included short stature $(\mathrm{n}=9 ; 64 \%)$, congenital cardiac abnormalities $(\mathrm{n}=7$, $54 \%)$, cardiomyopathy $(\mathrm{n}=4,33 \%)$ posteriorly rotated and/or low set ears $(\mathrm{n}=7 ; 58 \%)$, hypertelorism $(\mathrm{n}=8$; $67 \%)$, ptosis $(\mathrm{n}=8,62 \%)$, widely spaced nipples $(\mathrm{n}=7,78 \%)$ and epicanthal folds $(\mathrm{n}=6 ; 50 \%)$. Downslanting palpebral fissures $(n=3 ; 23 \%)$, triangular face $(n=4 ; 33 \%)$, short or webbed neck $(n=3,27 \%)$ and scoliosis $(\mathrm{n}=2,22 \%)$ were less often present. Distinguishing features of the CSCF patients are the wide nose with bulbous tip $(n=9 ; 82 \%)$, peri-orbital fullness $(n=7,64 \%)$ and the full cheeks $(n=9 ; 69 \%)$. Information about the neurological phenotype was available for 7 patients, who showed normal intelligence; three had autism 
spectrum disorder. Hypotonia was found in 7 patients (70\%), and joint laxity in 8 (80\%). The resemblance of CSCF to NS made us wonder if CSCF should also be considered a RASopathy; however, the RAS/MAPK pathway is downregulated instead of upregulated, suggesting an alternative explanation for the resemblance of CSCF to NS. Despite these molecular results, CSCF should be considered in the differential diagnosis of NS-patients based on the described clinical features.

Both our FMD2 patients showed phenotypes compatible with previously reported patients (Wade et al., 2017). Interestingly, our adult male FMD2 patient initially presented with ambiguous genitalia and he has a mild skeletal phenotype with normal/high intelligence. The pediatric patient with the recurrent variant for FMD2 has a severe cardiac, neurological and skeletal phenotype. Distinguishing features of the FMD2 patients in comparison to NS and CSCF were prominent supraorbital ridges, flexion contractures of the elbows, ulnar deviation of the hands, interphalangeal joint contractures, camptodactyly and keloid scarring (all present in both patients). Overlapping features with NS were short stature, congenital cardiac defects, hypertelorism, epicanthal folds, ptosis $(\mathrm{n}=1)$ and failure to thrive in infancy. Neither patient had a triangular face, short or webbed neck. FMD2 patients have more severe dysmorphism than CSCF patients (Figure 3 ) .

Regarding the occurrence of left sided heart lesions and the potential progressive aspect in our patient of aortic dilatation, these features go along with previous reports that genes which belong to TGF $\beta$ cascade are potentially prone to this subcategory of cardiac involvement (Baban et al., 2018). It might be wise to establish a specific screening program through serial electrocardiography and echocardiography detect progressive aortic dilatation or arrhythmias.

Our cohort in combination with the MAP3K 7 mutations causing FMD2 described in literature allowed for the first time to search for genotype/phenotype correlations, i.e. side-by-side comparison of the different missense mutations causing either FMD2 or CSCF. Confirming previous reports, we found that the FMD2causing mutations in $M A P 3 K^{\gamma}$ produce a gain-of-function effect on MAP3K7. Additionally, we now provide clear evidence that the CSCF-causing variants in $M A P 3 K^{7}$ have a loss-of-function effect. This is most clearly seen when looking at the expression and pThr187 levels of MAP3K7. Interestingly, when assessing one of the downstream targets of MAP3K7, NFkB (Xu and Lei, 2020), this distinction between the FMD2- or CSCF-causing variants is not evident, suggesting other molecular pathways are involved in the pathogenesis of these two disorders. Indeed, we have only assessed the effect on this pathway in co-expression of TAB1 and MAP3K7. Possibly there is a more distinct phenotype when assessing the function of MAP3K7 when in complex with TAB2/3. Additionally, other downstream pathways remain to be studied.

In this paper we significantly expanded the cohort of patients with either CSCF or FMD2 and performed for the first time a side-by-side comparison of the different missense variants related to either FMD2 or CSCF. We have shown that both clinical phenotypes exhibit a clear overlap with other syndromic connective tissue disorders with respect to bone deformities, short stature, hypermobile joints, contractures and cardiac anomalies. This fits what is known about MAP3KY's effect on the TGF $\beta$ pathway

(Le Goff et al., 2016; Yu et al., 2014). Additionally, we have identified that cardiac anomalies are common in patients with pathogenic MAP3Kr variants, which may be severe. The cardiac phenotype ranged from left sided congenital heart defects to cardiomyopathies (both hypertrophic and dilated) and arrhythmias. The latter subgroup is infrequent in NS (Pierpont and Digilio, 2018; Gelb et al., 2015). In conclusion, we show that expression and autophosphorylation levels of $M A P 3 K^{7}$ can serve as a molecular fingerprint to distinguish between FMD2- or CSCF-causing MAP3K 7 variants. Additionally, we show that MAP3K 7 variants should be considered in the differential diagnosis of patients with syndromic congenital cardiac (valve) anomalies and/or cardiomyopathy, syndromic connective tissue disorders as well as NS.

\section{Acknowledgements}

We thank our patients for their willingness to share their information to further our understanding of CSCF and FMD2. We thank dr. Ineke van der Burgt (Division of Human Genetics, Radboud medical center, Radboud University, Nijmegen the Netherlands) for her valuable insights and help setting up the study. 


\section{Conflict of interest}

None of the co-authors have any conflict of interest to declare.

\section{Data availability}

All data is available upon reasonable request.

\section{Bibliography}

Aashaq, S, Batool, A, Andrabi, KI. 2019. TAK1 mediates convergence of cellular signals for death and survival. Apoptosis 24: 3-20.

Baban, A, Magliozzi, M, Loeys, B, Adorisio, R, Alesi, V, Secinaro, A, Corica, B, Vricella, L, Dietz, HC, Drago, F, Novelli, A, Amodeo, A. 2018. First evidence of maternally inherited mosaicism in TGFBR1 and subtle primary myocardial changes in Loeys-Dietz syndrome: a case report. BMC Med. Genet. 19: 170-7.

Basart, H, van de Kar, A, Adès, L, Cho, T-J, Carter, E, Maas, SM, Wilson, LC, van der Horst, CMAM, Wade, EM, Robertson, SP, Hennekam, RC. 2015. Frontometaphyseal dysplasia and keloid formation without FLNA mutations. Am. J. Med. Genet. 167: 1215-1222.

Costantini, A, Wallgren-Pettersson, C, Mäkitie, O. 2018. Expansion of the clinical spectrum of frontometaphyseal dysplasia 2 caused by the recurrent mutation p.Pro485Leu in MAP3K7. European Journal of Medical Genetics 61: 612-615.

Dai, L, Aye Thu, C, Liu, X-Y, Xi, J, Cheung, PCF. 2012. TAK1, more than just innate immunity. IUBMB Life 64: 825-834.

Forney, WR, Robinson, SJ, Pascoe, DJ. 1966. Congenital heart disease, deafness, and skeletal malformations: a new syndrome? J Pediatr 68: 14-26.

Gelb, BD, Roberts, AE, Tartaglia, M. 2015. Cardiomyopathies in Noonan syndrome and the other RASopathies. Prog Pediatr Cardiol 39: 13-19.

Giuliano, F, Collignon, P, Paquis-Flucklinger, V, Bardot, J, Philip, N. 2005. A new three-generational family with frontometaphyseal dysplasia, male-to-female transmission, and a previously reported FLNA mutation. Am. J. Med. Genet. 132A: 222-222.

Jorge, AAL, Malaquias, AC, Arnhold, IJP, Mendonca, BB. 2009. Noonan syndrome and related disorders: a review of clinical features and mutations in genes of the RAS/MAPK pathway. Horm Res 71: 185-193.

Le Goff, C, Rogers, C, Le Goff, W, Pinto, G, Bonnet, D, Chrabieh, M, Alibeu, O, Nistchke, P, Munnich, A, Picard, C, Cormier-Daire, V. 2016. Heterozygous Mutations in MAP3K7, Encoding TGF- $\beta$-Activated Kinase 1, Cause Cardiospondylocarpofacial Syndrome. Am. J. Hum. Genet. 99: 407-413.

Micale, L, Morlino, S, Biagini, T, Carbone, A, Fusco, C, Ritelli, M, Giambra, V, Zoppi, N, Nardella, G, Notarangelo, A, Schirizzi, A, Mazzoccoli, G, Grammatico, P, Wade, EM, Mazza, T, Colombi, M, Castori, M. 2020. Insights into the molecular pathogenesis of cardiospondylocarpofacial syndrome: MAP3K7 c.737$7 \mathrm{~A}>\mathrm{G}$ variant alters the TGF $\beta$-mediated $\alpha$-SMA cytoskeleton assembly and autophagy. Biochim Biophys Acta Mol Basis Dis 1866: 165742.

Morlino, S, Castori, M, Dordoni, C, Cinquina, V, Santoro, G, Grammatico, P, Venturini, M, Colombi, M, Ritelli, M. 2018. A novel MAP3K7 splice mutation causes cardiospondylocarpofacial syndrome with features of hereditary connective tissue disorder. Eur J Hum Genet: 1-5.

Pierpont, ME, Digilio, MC. 2018. Cardiovascular disease in Noonan syndrome. Current Opinion in Pediatrics 30: $601-608$.

Proietti Onori, M, Koopal, B, Everman, DB, Worthington, JD, Jones, JR, Ploeg, MA, Mientjes, E, van Bon, BW, Kleefstra, T, Schulman, H, Kushner, SA, Küry, S, Elgersma, Y, van Woerden, GM. 2018. The 
intellectual disability-associated CAMK2G p.Arg292Pro mutation acts as a pathogenic gain-of-function. Hum. Mutat. 39: 2008-2024.

Robertson, SP. 2004. Molecular pathology of filamin A: diverse phenotypes, many functions. Clinical Dysmorphology 13: 123-131.

Sakurai, H, Miyoshi, H, Mizukami, J, Sugita, T. 2000. Phosphorylation-dependent activation of TAK1 mitogen-activated protein kinase kinase kinase by TAB1. FEBS Lett. 474: 141-145.

Sobreira, N, Schiettecatte, F, Valle, D, Hamosh, A. 2015. GeneMatcher: a matching tool for connecting investigators with an interest in the same gene. Hum. Mutat. 36: 928-930.

Sousa, SB, Baujat, G, Abadie, V, Bonnet, D, Sidi, D, Munnich, A, Krakow, D, Cormier-Daire, V. 2010. Postnatal growth retardation, facial dysmorphism, spondylocarpal synostosis, cardiac defect, and inner ear malformation (cardiospondylocarpofacial syndrome?)-a distinct syndrome? Am. J. Med. Genet. 152A: 539546.

van der Burgt, I. 2007. Noonan syndrome. Orphanet Journal of Rare Diseases 2: 4-6.

Wade, EM, Daniel, PB, Jenkins, ZA, McInerney-Leo, A, Leo, P, Morgan, T, Addor, MC, Adès, LC, Bertola, D, Bohring, A, Carter, E, Cho, T-J, Duba, H-C, Fletcher, E, Kim, CA, Krakow, D, Morava, E, Neuhann, T, Superti-Furga, A, Veenstra-Knol, I, Wieczorek, D, Wilson, LC, Hennekam, RCM, Sutherland-Smith, AJ, Strom, TM, Wilkie, AOM, Brown, MA, Duncan, EL, Markie, DM, Robertson, SP. 2016. Mutations in MAP3K7 that Alter the Activity of the TAK1 Signaling Complex Cause Frontometaphyseal Dysplasia. Am. J. Hum. Genet. 99: 392-406.

Wade, EM, Jenkins, ZA, Daniel, PB, Morgan, T, Addor, MC, Adès, LC, Bertola, D, Bohring, A, Carter, E, Cho, T-J, de Geus, CM, Duba, H-C, Fletcher, E, Hadzsiev, K, Hennekam, RCM, Kim, CA, Krakow, D, Morava, E, Neuhann, T, Sillence, D, Superti-Furga, A, Veenstra-Knol, HE, Wieczorek, D, Wilson, LC, Markie, DM, Robertson, SP. 2017. Autosomal dominant frontometaphyseal dysplasia: Delineation of the clinical phenotype. Am. J. Med. Genet.

Xu, Y-R, Lei, C-Q. 2020. TAK1-TABs Complex: A Central Signalosome in Inflammatory Responses. Front Immunol 11: 608976.

Yu, J, Zhang, F, Wang, S, Zhang, Y, Fan, M, Xu, Z. 2014. TAK1 is activated by TGF- $\beta$ signaling and controls axonal growth during brain development. J Mol Cell Biol 6: 349-351.

Table 1. Patient characteristics and detailed phenotypic description of all included patients.

Figure legends

Figure 1. Schematic overview of MAP3K7 showing the localization of the different variants. Schematic overview of $M A P 3 K^{\gamma}$ showing the Kinase and TAB1 binding domain at the N-terminal and the TAB2/3 binding domain at the C-terminal of MAP3K 7 . In black the CSCF-related MAP3K 7 variants are indicated and in blue the FMD2-related $M A P 3 K^{7}$ variants. The underlined variants are the novel variants identified in our cohort. MAP3K $7^{\mathrm{P} 485 \mathrm{~L}}$ is the most recurrent FMD2-causing variant.

Figure 2. Light photographs of patients with CSCF

Five patients with CSCF: patients show hypertelorism, full eyelids, full cheeks, round nasal tip, low set ears and almond shaped eyes.

Figure 3. Clinical description and light photography/radiological pictures of the patient with the recurrent P485L variant causing FMD2 (patient 16).

Cranio-facial dysmorphisms at 2 months (panels 1-3): bi-temporal narrowing, hypotelorism, down-slanting palpebral fissures, short nose with flat nasal bridge and depressed root, long and deeply grooved filter, microretrognathia. Radiological findings (panels 4-7) at 7 months: a partial fusion of cervical vertebrae 
(C2-C3) and reduced bone length in relation to age. Magnetic resonance imaging (MRI) at 7 months: T2weighted hyperintensity of presumed vascular lesion in the coronal and transverse planes (panels 8-9); T1 weighed scans in the sagittal plane: cerebral atrophy, reduced corpus callosum, and Chiari 1 malformation (panel 10). Cranial time-of-flight (TOF) MR angiogram (3T) at 7 months: flow reduction of the M2 branch of left middle cerebral artery (MCA) (panel 11). Facial appearance at 3 y.o. (panel 12).

Figure 4. Differential expression and autophosphorylation of variants in MAP3K7 causing CSCF or FMD2. A) Western blot analysis showing reduced expression levels of CSCF-causing MAP3K 7 variants (black circles), compared to MAP3K 7 WT, but normal or even increased expression levels of FMD2-causing MAP3K 7 variants (blue circles), compared to MAP3K7 ${ }^{\mathrm{WT}}$, when overexpressed in HEK293T cells. $\mathrm{N}=3$ for all $\mathrm{CSCF}$-causing variants, except MAP3K $77^{\mathrm{W} 241 \mathrm{G}}(\mathrm{N}=6) . \mathrm{N}=6$ for all FMD2-causing variants, except MAP3K $7^{\mathrm{Y} 113 \mathrm{D}}$ and MAP3K7 $7^{\mathrm{G} 168 \mathrm{R}}(\mathrm{N}=3)$. WT $\mathrm{N}=7$. B) Western blot analysis showing that expression levels of CSCF-causing MAP3K 7 variants is normalized upon TAB1 co-expression, except for MAP3K7 ${ }^{\mathrm{W} 241 \mathrm{G}}$. $\mathrm{N}=7$ for all conditions, except for $\mathrm{WT}(\mathrm{N}=14)$ and MAP3K $7^{\mathrm{W} 241 \mathrm{G}}(\mathrm{N}=6)$. C) Western blot analysis of the autophosphorylation of MAP3K 7 at Thr187 showing that except for MAP3K $7^{\mathrm{R} 83 \mathrm{H}}$, all CSCF-causing MAP3K7 variants show reduced pThr187 compared to MAP3K $7{ }^{\mathrm{WT}}$, whereas FMD2-causing variants show normal or increased levels of pThr187 compared to MAP3K $7{ }^{\mathrm{WT}}$. $\mathrm{N}=7$ for all conditions, except for WT $(\mathrm{N}=14)$ and MAP3K $7^{\mathrm{Y} 206 \mathrm{C}}$ and MAP3K7 ${ }^{\mathrm{Y} 113 \mathrm{D}}(\mathrm{N}=6)$. Error bars represent standard error of the mean (SEM). ${ }^{*} \mathrm{p}<0.05 ;{ }^{* *} \mathrm{p}<0.01 ; * * * \mathrm{p}<0.0001$.

Figure 5. Western blot analysis of downstream targets of MAP3K7. A) Western blot analysis reveals reduced phosphorylation of $\mathrm{NFkB}$ upon overexpression of most CSCF-causing (black circles) and some FMD2-causing (blue circles) MAP3K7 variants in HEK-293T cells, compared to MAP3K7 ${ }^{\mathrm{WT}}$. $\mathrm{N}=7$ for all conditions except for WT $(\mathrm{N}=10)$. B) Western blot analysis of phosphoERK1/2 reveals reduced phosphorylation of ERK1/2 upon overexpression of most CSCF-causing MAP3K 7 variants compared to MAP3K7 ${ }^{\mathrm{WT}}$, except for MAP3K7 ${ }^{\mathrm{R} 83 \mathrm{H}}$. $\mathrm{N}=4$ for all conditions, except for $\mathrm{WT}(\mathrm{N}=8)$. Error bars represent standard error of the mean (SEM). ${ }^{*} \mathrm{p}<0.05 ;{ }^{* * *} \mathrm{p}<0.0001$.

\section{Hosted file}

Table 1.docx available at https://authorea.com/users/447164/articles/546219-involvement-ofmap3k7-in-fmd2-and-cscf-delineation-of-genotype-phenotype-correlations

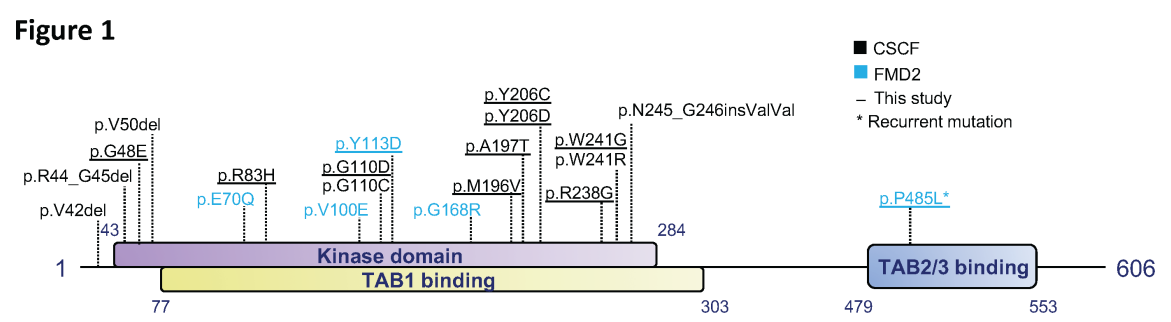


Figure 2

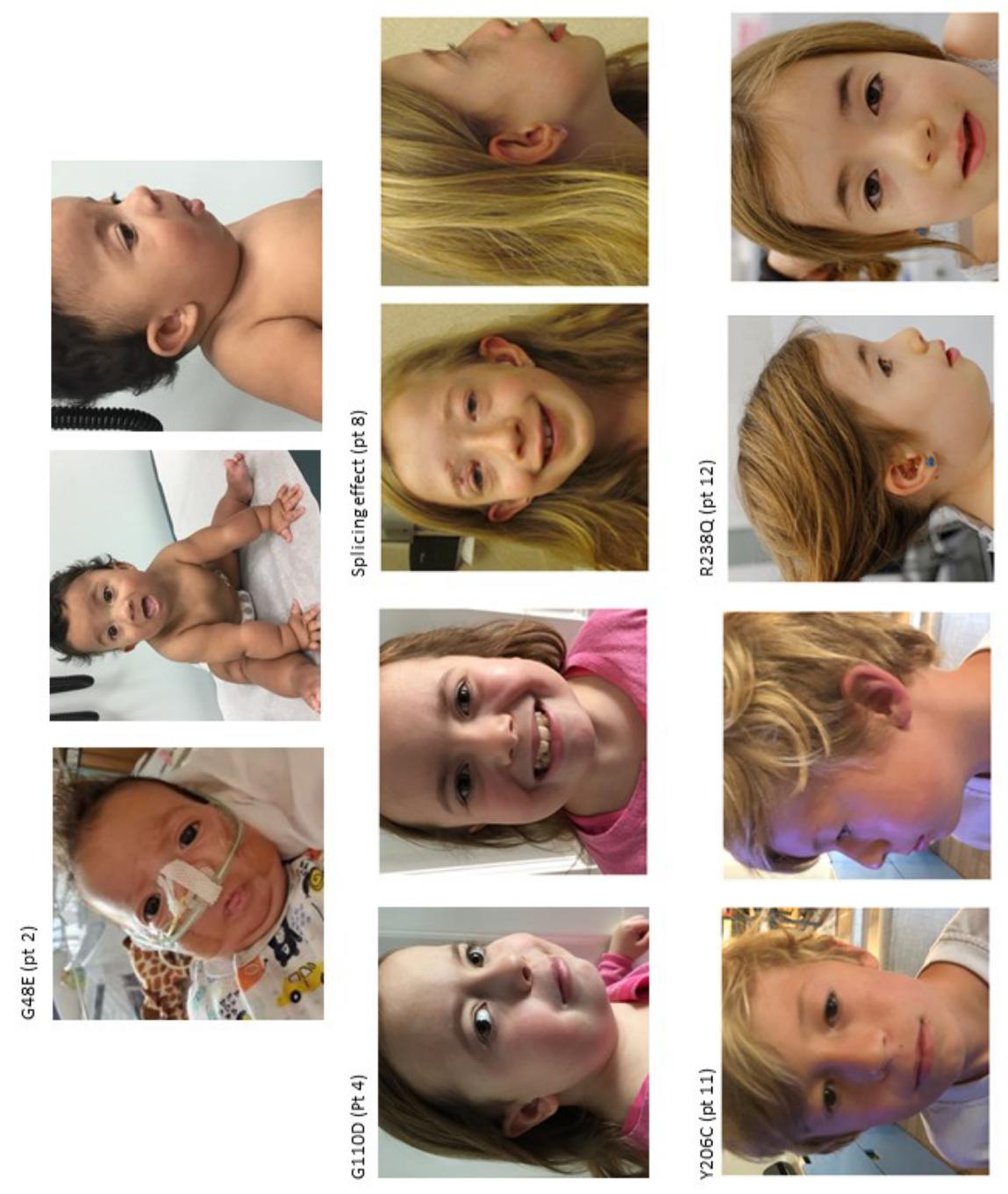


Figure 3
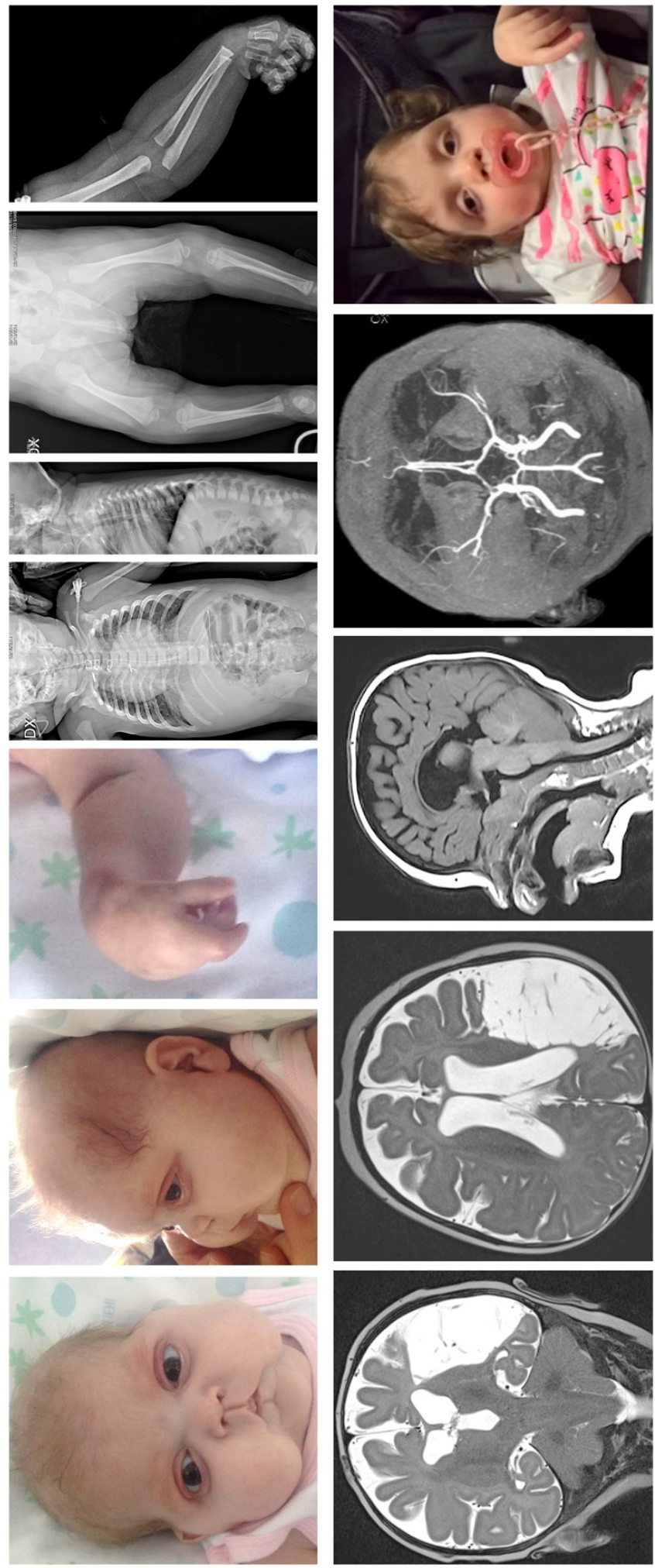
Figure 4

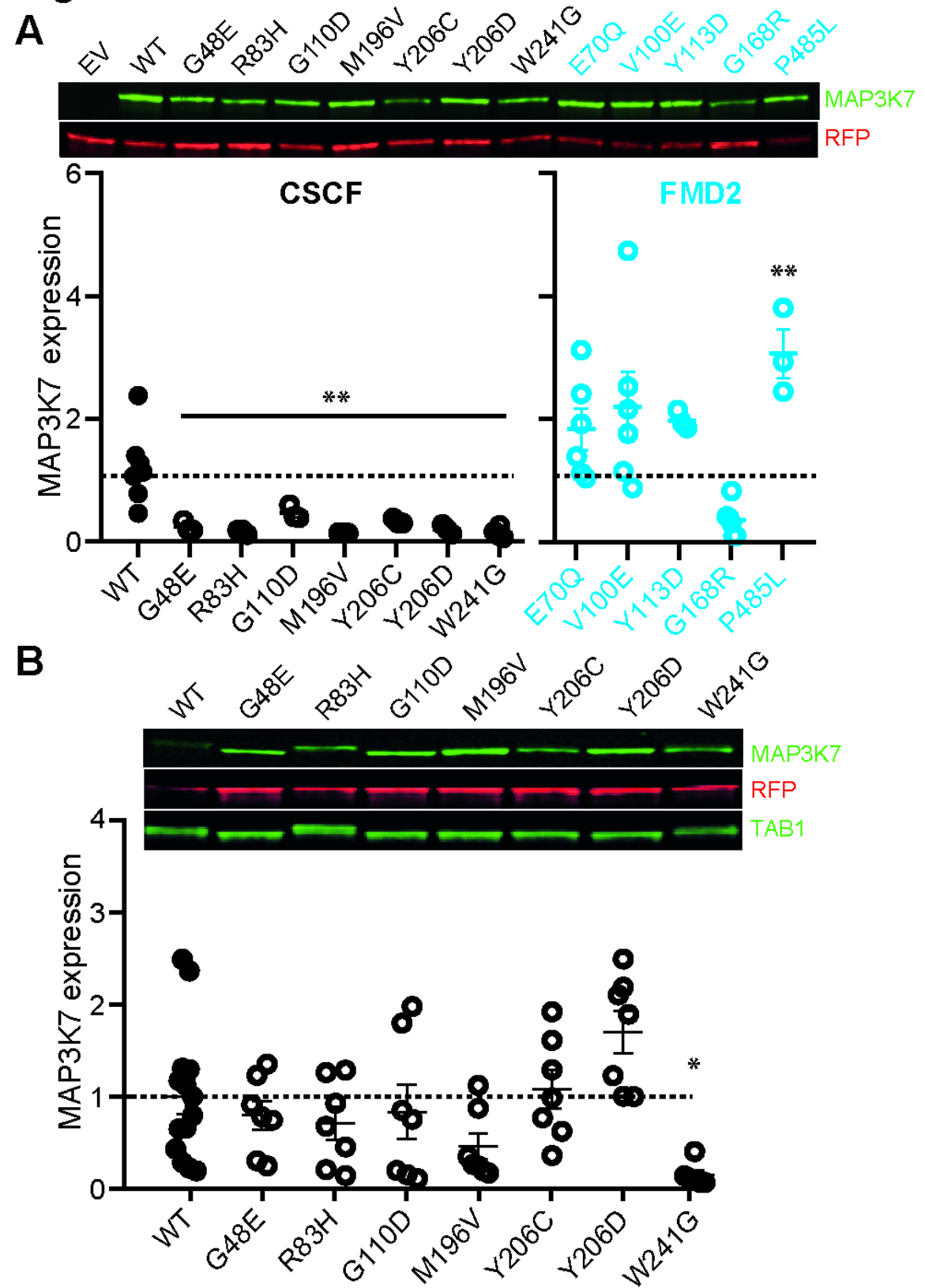

C

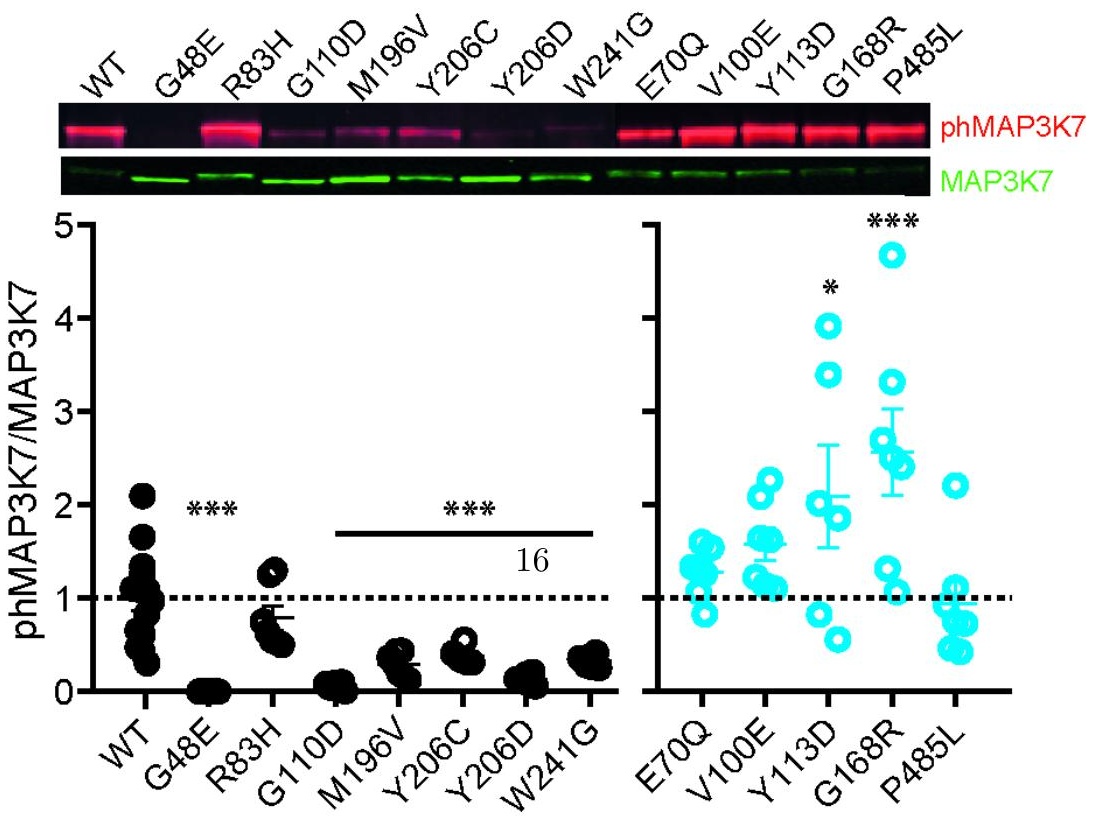


Figure 5
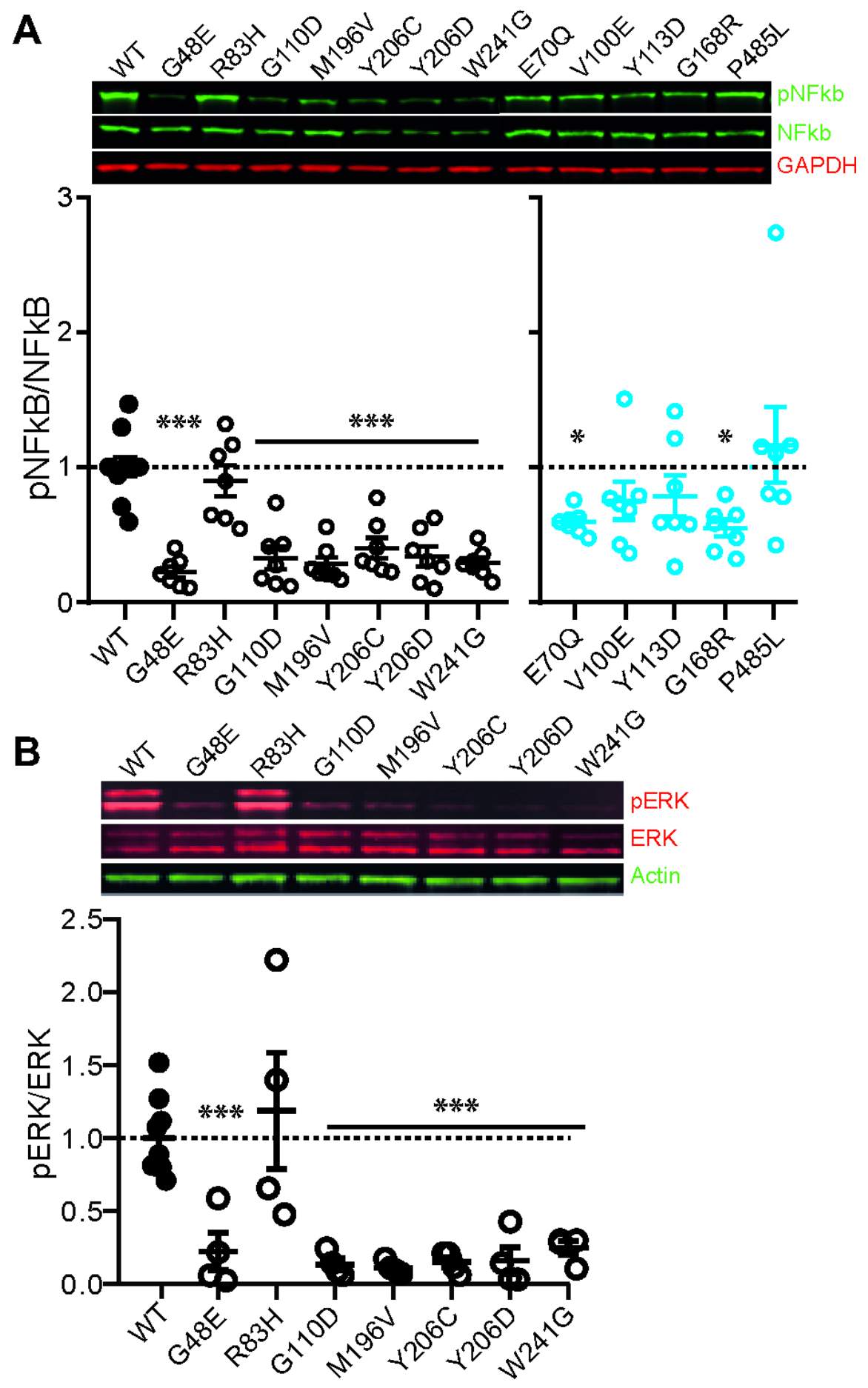